\title{
Effects of exercise timing on sleep architecture and nocturnal blood pressure in prehypertensives
}

\author{
This article was published in the following Dove Press journal: \\ Vascular Health and Risk Management \\ 12 December 2014 \\ Number of times this article has been viewed
}

\author{
Kimberly Fairbrother' \\ Ben Cartner' \\ Jessica R Alley' \\ Chelsea D Curry' \\ David L Dickinson ${ }^{2}$ \\ David M Morris' \\ Scott R Collier ${ }^{\prime}$ \\ 'Vascular Biology and Autonomic \\ Studies Laboratory, Department \\ of Health and Exercise Science, \\ ${ }^{2}$ Department of Economics, \\ Appalachian State University, \\ Boone, NC, USA
}

Correspondence: Scott R Collier Vascular Biology and Autonomic Studies Laboratory, Appalachian State University, I I I Rivers Street - HCC 046, Boone, NC 28608, USA

$\mathrm{Tel}+|8282627| 45$

$\mathrm{Fax}+\mid 8282623138$

Email colliersr@appstate.edu
Background: During nocturnal sleep, blood pressure (BP) "dips" compared to diurnal BP, reducing stress on the cardiovascular system. Both the hypotensive response elicited by acute aerobic exercise and sleep quality can impact this dipping response.

Purpose: The purpose of this study was to investigate the effects of aerobic exercise timing on circadian BP changes and sleep architecture.

Materials and methods: Twenty prehypertensive subjects completed the study. During four test sessions, participants first completed a graded exercise test to exhaustion and then performed 30 minutes of treadmill exercise at 7 am (7A), 1 pm (1P), and $7 \mathrm{pm}(7 \mathrm{P})$ in a random, counterbalanced order at $65 \%$ of the heart rate obtained at peak oxygen uptake. An ambulatory cuff was used to monitor BP responses during 24 hours following exercise, and an ambulatory sleep-monitoring headband was worn during sleep following each session.

Results: Aerobic exercise at 7A invoked a greater dip in nocturnal systolic BP than exercise at $1 \mathrm{P}$ or $7 \mathrm{P}$, although the greatest dip in nocturnal diastolic BP occurred following 7P. Compared to $1 \mathrm{P}, 7 \mathrm{~A}$ also invoked greater time spent in deep sleep.

Conclusion: These data indicate that early morning may be the most beneficial time to engage in aerobic exercise to enhance nocturnal BP changes and quality of sleep.

Keywords: nocturnal dipping, prehypertension, aerobic exercise

\section{Introduction}

Hypertension is a significant modifiable risk factor associated with stroke, coronary artery disease, end-stage renal disease, and congestive heart failure. ${ }^{1,2}$ The risk of cardiovascular disease (CVD) increases as blood pressure (BP) rises above $115 / 75 \mathrm{mmHg}$, and this risk doubles with each $20 / 10 \mathrm{mmHg}$ increase. ${ }^{3}$ Normal circadian variation in resting BP is characterized by a nocturnal decrease of $10 \%-20 \%$ of daytime levels in the majority of normotensive and hypertensive individuals. ${ }^{4,5}$ This lowering of BP during the night has been suggested to be a restorative physiological process. ${ }^{6}$ Individuals whose nocturnal BP does not decrease by more than $10 \%$ are referred to as "nondippers". ${ }^{5}$ Nondipping has been documented in patients with sleep apnea, advanced kidney disease, nocturia, and increased sympathetic activation. ${ }^{7}$ Nondipping is a significant risk factor for left ventricular hypertrophy and CVD regardless of 24-hour BP normality $(<135 / 80 \mathrm{mmHg}){ }^{8,9}$ Furthermore, a significant association exists between poor sleep quality and nondipping status in prehypertensive patients $;{ }^{4}$ in one study, ${ }^{4}$ those with newly diagnosed hypertension and also classified as poor sleepers exhibited almost a 3-fold increase in risk of being a nondipper. 
Inadequate sleep, specifically less than 6-7 quality hours per night, has been correlated with clinical manifestations, such as hypertension, ${ }^{10,11}$ stroke, ${ }^{12}$ obesity, ${ }^{11}$ and ultimately an increased risk of mortality. Sleep deprivation has a direct impact on hypertension and increased sympathetic nervous activity, ${ }^{13}$ independently of its influence on body weight. Even after adjusting for sex and obesity, poor sleep efficiency was associated with a $4 \mathrm{mmHg}$ increase in systolic BP (SBP). ${ }^{10}$ Poor sleep has also been associated with a 2.5-fold increase in odds for developing prehypertension (SBP 120-139 and diastolic BP [DBP] 80-89 mmHg). ${ }^{14}$ Therefore, poor sleep quality may be a modifiable risk factor for hypertension and CVD.

It has been shown that daytime exercise exerts a positive effect on nighttime sleep quality. ${ }^{15}$ The National Sleep Foundation recommends exercise as a nonpharmacological intervention for improving sleep quality. Sleep researchers have predicted that duration and quality of sleep are higher in physically fit individuals, especially on nights following exercise perturbations. ${ }^{16}$ In a meta-analysis by Kubitz et al, ${ }^{16}$ it was reported that exercise increased total sleep time as well as time in deep sleep and decreased sleep-onset latency (SOL). Previous studies have documented that aerobic exercise, specifically, is associated with decreases in light-sleep stages as well, as with increases in the deeper, most restorative stages of sleep. ${ }^{17}$

Aerobic exercise is currently recommended as a modality for decreasing cardiovascular risk by decreasing resting BP. An acute, moderate-intensity aerobic exercise bout elicits a decrease in BP known as postexercise hypotension (PEH), which resets the baroreflex to lower operating pressures. ${ }^{18}$ These lower BPs are observed for up to 24 hours postexercise in hypertensive individuals, ${ }^{3}$ lending clinical effectiveness to aerobic exercise. The duration and magnitude of the $\mathrm{PEH}$ response may depend on the time of day of exercise due to the circadian variation of $\mathrm{BP},{ }^{19}$ although there is limited research examining this phenomenon. Park et $\mathrm{al}^{2}$ investigated BP responses following acute bouts of exercise in both the morning and afternoon hours. Exercise in the afternoon elicited a greater PEH response in medicated hypertensive nondippers. Clinically, exercise is a nonpharmacological treatment for lowering BP and reducing the risk of CVD; however, the timing of exercise may contribute to more effective results following the exercise bout.

No previous studies have compared the effects of exercise timing on sleep architecture concomitantly with nocturnal BP variations. Therefore, the purpose of our investigation was to examine how aerobic exercise timing affects the quality of sleep and diurnal BP variations in young, prehypertensive individuals. We hypothesized that exercise at $7 \mathrm{pm}$ (7P) would elicit more favorable changes in sleep architecture and nocturnal BP responses than exercise at 7 am (7A) or $1 \mathrm{pm}(1 \mathrm{P})$, due to greater time spent in $\mathrm{PEH}$.

\section{Materials and methods Subjects}

An a priori power calculation with an $\alpha$-level of 0.05 with 20 subjects in the model yielded a power of 0.91 to detect a medium-size effect (G*Power, version 3.1.3; Uni Kiel, Germany). Therefore, 20 prehypertensive men and women (SBP range of 120-139, DBP $80-89 \mathrm{mmHg}$ ) between the ages of 30 years and 60 years were recruited from the local community. Subjects had no self-reported sleep disorders, were nonsmokers, and were not using any medications, including aspirin therapy or sleep aids, as identified in health and activity-history questionnaires. Subjects were classified as sedentary to recreationally active, with no more than one risk factor according to the American College of Sports Medicine's risk stratification. Investigators reviewed the screening questionnaires to ensure subjects met these required guidelines. The investigation was approved by the Institutional Review Board of Appalachian State University.

\section{Experimental design}

Subjects reported to the laboratory on four separate occasions. On the first visit, each subject completed a written informed consent as well as the American College of Sports Medicine physical activity/health-history questionnaire. Age, height, weight, and BP were recorded, followed by an assessment of maximal aerobic capacity. Each subject performed a graded treadmill-exercise test to volitional exhaustion, while expired air was collected and analyzed to determine peak oxygen uptake ( $\mathrm{VO}_{2}$ peak). In an attempt to decrease the effects of experimental stress on ambulatory BP values, subjects were familiarized with all of the data-collection equipment utilized during the study prior to the second visit and were instructed to take the sleep-monitoring device home and wear it prior to the first night of data collection.

During visits two, three, and four, participants completed the aerobic exercise protocol at 7A, 1P, and 7P in a randomized order that was determined using a random-number generator. An average washout period of 72 hours was allotted between subsequent exercise sessions, which all took place during a weekday (Monday, Thursday, Monday). Subjects were asked to refrain from alcohol and caffeine 12 hours prior to each exercise session and for the 24 hours following each 
session during which data collection occurred. Following each exercise session, each participant wore an ambulatory BP device (Oscar 2; SunTech Medical, Morrisville, NC, USA) for the next 24-hour period, as well as an ambulatory sleepmonitoring system (Personal Sleep Manager; Zeo, Newton, MA, USA) on the nights following exercise. Subjects were instructed to maintain their typical schedules, including same time to bed and time to wake, following each exercise bout in order to control for total sleep time.

\section{$\mathrm{VO}_{2}$ peak}

$\mathrm{VO}_{2}$ peak was assessed using a customized treadmill protocol (modified Balke protocol). Briefly, initial intensity started at $2.5 \mathrm{mph}(4.02 \mathrm{~km} / \mathrm{h})$ and increased by $1 \mathrm{mph}$ every 2 minutes until a comfortable pace was established, at which point the test subject exercised as the grade of the treadmill was increased $2.5 \%$ at 2-minute intervals until volitional fatigue was reached. Heart rate was recorded once per minute from the start of the protocol and a minimum of 4 minutes into recovery using a heart-rate monitor (Polar Electro, Woodbury, NY, USA). Ratings of perceived exertion were also assessed within the last 15 seconds of each stage. Expired gases were analyzed using a True One 2400 metabolic system (Parvo Medics, Sandy, UT, USA). Exercise capacity was assessed by exercise time and total workload expressed in metabolic equivalent of task. Maximal effort was considered reached when the subjects met three of the four following criteria: no change in heart rate with a change in workload, a final rating of perceived exertion of 17 , a respiratory exchange ratio $>1.14$, or volitional exhaustion. A 12-lead electrocardiogram was used to monitor the test for men and women greater than 45 years and 50 years of age, respectively.

\section{Exercise protocol}

Values attained from the preliminary exercise testing session were used to design the aerobic exercise prescription. All individuals performed a 30-minute aerobic exercise bout on a motorized treadmill at an intensity corresponding to $65 \%$ of their heart rate attained at $\mathrm{VO}_{2}$ peak. Heart rate was monitored throughout every 30-minute session using the
Polar heart-rate monitor to ensure subjects remained at their prescribed intensity.

\section{Ambulatory BP measurement}

Upon completion of each exercise session, subjects were outfitted with the Oscar 2 ambulatory BP device. The device was programmed to take oscillatory BP measurements every 20 minutes throughout the 24 -hour postexercise period and every 40 minutes during sleep. Data were stored within the device and uploaded onto the laboratory computer.

\section{Ambulatory sleep-stage measurement}

On nights following the completed exercise sessions, subjects wore an ambulatory sleep-monitoring headband. The system consisted of a soft headband and a bedside display. The two-piece system utilizes dry-sensor electroencephalography technology to transmit brainwave data wirelessly to the bedside display, where it can be stored for later analysis. The Zeo system has been validated against in-laboratory polysomnography for deep, rapid eye movement (REM), and total sleep time, and has been shown to be an accurate and comfortable technique to measure sleep stages. ${ }^{20}$ This system allows for subjects to maintain their typical sleeping habits in their own home environment, while permitting accurate sleep-stage measurements.

\section{Treatment of data}

A $1 \times 3$ repeated-measures analysis of variance with a Bonferroni post hoc test was employed to determine the differences between exercise time and each outcome variable. All data were analyzed by SPSS version 17 (SPSS, Chicago, IL, USA). All data are shown as means \pm standard error of the mean.

\section{Results}

Subject characteristics are shown in Table 1. The subjects were classified as prehypertensive (BP >120/80 mmHg) with a mean body mass index indicative of overweight $(>25)$. Each subject performed all of the exercise bouts at the predetermined time of day. In the nights following

Table I Subject characteristics

\begin{tabular}{llllll}
\hline $\begin{array}{l}\text { Age } \\
\text { (years) }\end{array}$ & $\begin{array}{l}\text { Height } \\
(\mathbf{c m})\end{array}$ & $\begin{array}{l}\text { Weight } \\
(\mathbf{k g})\end{array}$ & $\begin{array}{l}\text { BMI } \\
\left(\mathbf{k g} / \mathbf{m}^{2}\right)\end{array}$ & $\begin{array}{l}\text { VO } \text { peak } \\
(\mathbf{m L} / \mathbf{k g} / \mathbf{m i n})\end{array}$ & $\begin{array}{l}\text { BP } \\
(\mathbf{m m H g})\end{array}$ \\
\hline $41.9 \pm 3.2$ & $171.5 \pm 2.3$ & $75 \pm 4.5$ & $25 \pm 2.2$ & $38 \pm 3.2$ & $133 / 80 \pm 3.1 / 1.9$ \\
\hline
\end{tabular}

Note: Data are means \pm standard error of mean.

Abbreviations: $\mathrm{BMI}$, body mass index; $\mathrm{VO}_{2}$, oxygen uptake; $\mathrm{BP}$, blood pressure. 
each exercise bout, there were no significant differences in total sleep time and wake times for a given subject. This allowed for the comparison of absolute differences in the various components of sleep architecture among the separate exercise-timing bouts.

Mean nighttime SBP was significantly improved by the time of exercise $(P=0.05)$ (Figure 1A). Mean nighttime SBP was significantly reduced following the 7A time point $(107 \pm 4.8 \mathrm{mmHg})$ relative to $1 \mathrm{P}(114.8 \pm 5.2 \mathrm{mmHg}, P=0.043)$. Mean nighttime DBP was also significantly improved by the time of exercise $(P=0.038$ ) (Figure $1 \mathrm{~B})$, and was significantly lower following the 7A time point $(60.8 \pm 0.89 \mathrm{mmHg}) \mathrm{com}-$ pared to $1 \mathrm{P}(65.6 \pm 0.99 \mathrm{mmHg}, P=0.025)$. Mean nighttime DBP also improved between the $7 \mathrm{P}(61.8 \pm 0.98 \mathrm{mmHg})$ and the $1 \mathrm{P}(65.6 \pm 0.84 \mathrm{mmHg}, P=0.054)$ conditions.

A

Mean nighttime SBP

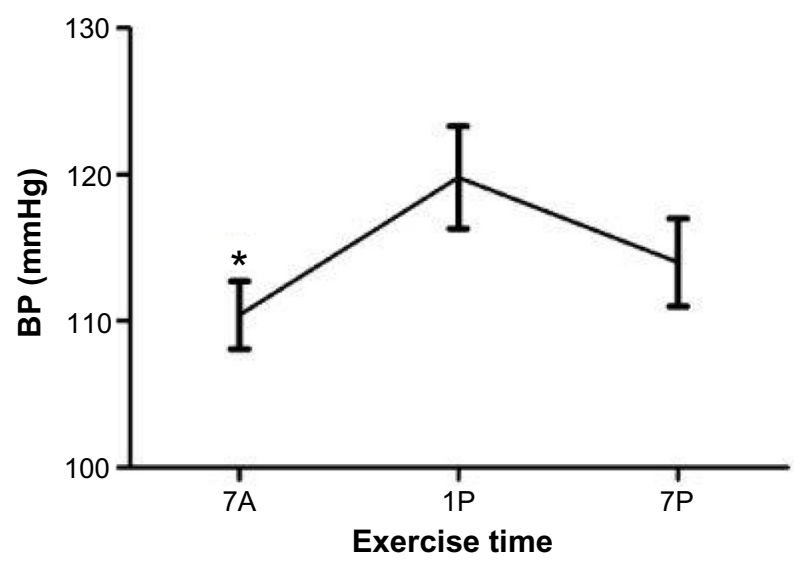

B Mean nighttime DBP

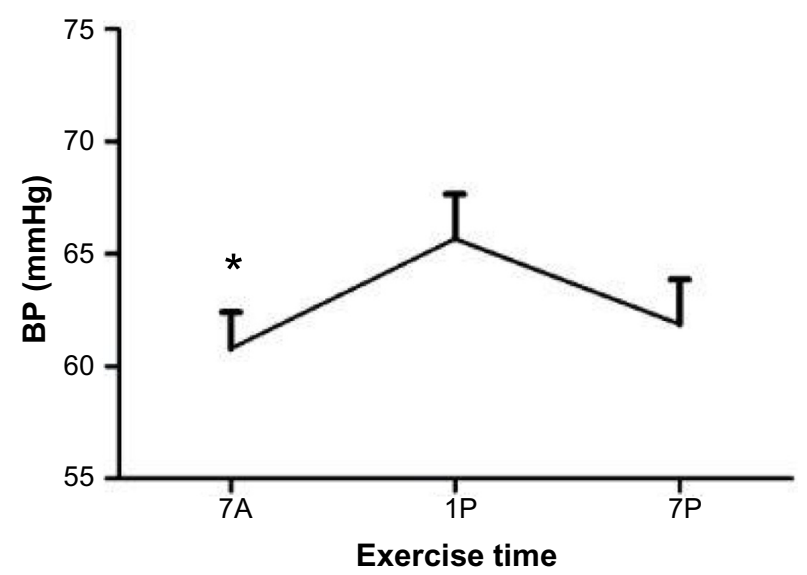

Figure I Nocturnal Blood Pressures.

Notes: (A) Mean nighttime systolic BP (SBP) and (B) diastolic BP (DBP) compared to each time-of-day exercise bout. Data are means \pm standard error of mean. *7A significantly different from IP $(P \leq 0.05)$.

Abbreviations: BP, blood pressure; 7A, 7 am exercise condition; IP, I pm exercise condition; 7P, 7 pm exercise condition.
The percentage of nocturnal BP dipping was significantly altered by exercise for both systolic and diastolic dipping ( $P=0.034$ and $P=0.031$, respectively) (Figure 2 ). The percentage drop in SBP was significantly larger after the 7A time point (16.67\% $\pm 0.93 \%)$ compared to $1 \mathrm{P}(12.3 \% \pm 1.2 \%$; $P=0.009)$. The dip in DBP was also more profound following $7 \mathrm{~A}(20.9 \% \pm 1.15 \%)$ compared to $1 \mathrm{P}(15.4 \% \pm 1.6 \%$; $P=0.03)$ exercise, although the DBP dip was greatest and significantly different following $7 \mathrm{P}(21.1 \% \pm 1.9 \%)$ compared to $1 \mathrm{P}(P=0.03)$ exercise.

Time spent in deep sleep was significantly altered by the timing of exercise $(P=0.008)$ (Figure 3$)$. More time was spent in deep sleep following 7A (63.3 \pm 6.1 minutes) versus $1 \mathrm{P}$ exercise $(43 \pm 3.19$ minutes, $P=0.008)$. Time spent in REM sleep was also significantly altered by the time of exercise $(P=0.038)$ (Figure 3$)$. The bout of exercise at $7 \mathrm{~A}$ presented significantly less time spent in REM sleep (98.2 \pm 12.9 minutes) than at $1 \mathrm{P}$ (117.4 \pm 12.4 minutes, $P=0.05)$. Exercise timing had no significant effects on time spent in light sleep, although it followed a comparable trend (Table 2).

Exercise timing also significantly altered SOL $(P=0.001)$, with $7 \mathrm{~A}$ resulting in decreased time to fall asleep (11.2 \pm 2.4 minutes) compared to $1 \mathrm{P}(32.8 \pm 4.2$ minutes, $P=0.001)$ or $7 \mathrm{P}(21.3 \pm 3$ minutes, $P=0.001)$ (Figure 4). Partaking in the $7 \mathrm{P}$ exercise bout also elicited less time to sleep onset than $1 \mathrm{P}(P=0.002)$. Awakenings after the onset of sleep were significantly altered with exercise timing $(P=0.018)$ (Table 2). Following 7A exercise, there was a decreased number of awakenings $(3.3 \pm 0.6)$ when compared to $1 \mathrm{P}(5.9 \pm 0.9, P=0.021)$ and $7 \mathrm{P}(5.5 \pm 1.1, P=0.016)$. In addition, time spent awake after the onset of sleep was sig-

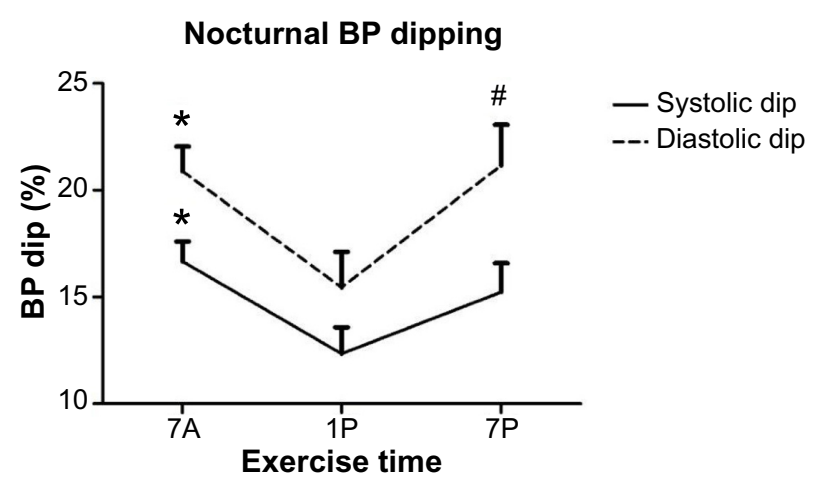

Figure 2 Systolic and diastolic blood pressure (BP) dipping compared to each timeof-day exercise bout. Data are means \pm standard error of mean.

Notes: $* 7 A$ significantly different from IP $(P \leq 0.05)$. ${ }^{* 7 P}$ significantly different than IP $(P<0.05)$.

Abbreviations: 7A, 7 am exercise condition; IP, I pm exercise condition; 7P, 7 pm exercise condition. 


\section{Deep and REM sleep}

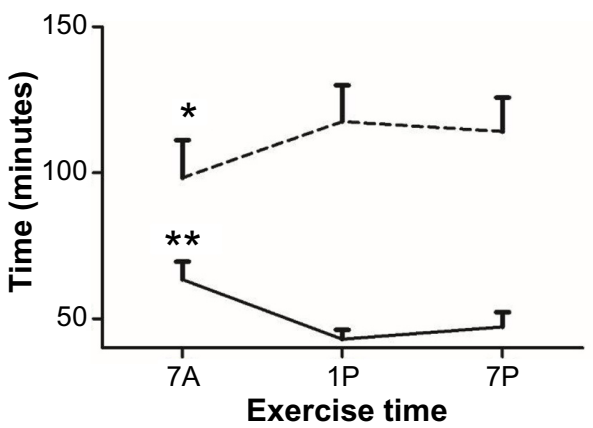

Figure 3 Time spent in deep and rapid eye movement (REM) sleep compared to each time-of-day exercise bout. Data are means \pm standard error of mean.

Notes: $* 7 A$ significantly different from IP ( $P \leq 0.05)$; **7A significantly different from IP $(P \leq 0.01)$.

Abbreviations: 7A, 7 am exercise condition; IP, I pm exercise condition; 7P, 7 pm exercise condition.

nificantly lower in 7A (15.8 \pm 5.0 minutes $)$ compared to $1 \mathrm{P}$ or 7P (29.0 \pm 5.9 and 24.6 \pm 4.9 minutes, respectively; $P=0.025)$ exercise, although time spent awake before the onset of sleep was not significantly different between exercise time points (Table 2).

\section{Discussion}

The present study investigated the effects of aerobic exercise timing on circadian BP variations and sleep architecture. Contrary to our hypothesis, the main finding was that a bout of moderate-intensity aerobic exercise at 7A resulted in the most beneficial responses in BP and improved overall sleep quality compared to $1 \mathrm{P}$ or $7 \mathrm{P}$. Exercise completed at the $7 \mathrm{~A}$ time point evoked a greater drop in nocturnal BP and elicited more time spent in deep sleep, less time spent in REM sleep, shorter SOL, and a decreased number of awakenings following the onset of sleep.

This study showed lower nighttime BP following 7A exercise that was not observed following exercise at $1 \mathrm{P}$ or 7P. Decreases in nighttime BP following 7A exercise may be attributed to a reduction in catecholamines, cortisol, and other stress hormones that are present at higher amounts during the waking hours. ${ }^{21,22}$ Many physiological

Table 2 Sleep variables compared to exercise conditions

\begin{tabular}{llll}
\hline Sleep variable & 7A & IP & 7P \\
\hline Wake time (minutes) & $16.4 \pm 3.0$ & $29.2 \pm 6.9$ & $21.0 \pm 4.5$ \\
Light sleep (minutes) & $260.2 \pm 15.5$ & $273.5 \pm 13.4$ & $268.0 \pm 1.0$ \\
Awakenings (n) & $3.3 \pm 0.6 *$ & $5.9 \pm 0.9$ & $5.5 \pm 1.1$ \\
Awakenings (minutes) & $15.8 \pm 5.0 *$ & $29.0 \pm 5.9$ & $24.6 \pm 4.9$ \\
\hline
\end{tabular}

Notes: *Significantly different from IP and 7P $(P<0.05)$. $P$ represents result of repeated-measures analysis of variance. Data are means \pm standard error of mean. Abbreviations: 7A, 7 am exercise condition; IP, I pm exercise condition; 7P, 7 pm exercise condition.

\section{Sleep-onset latency}

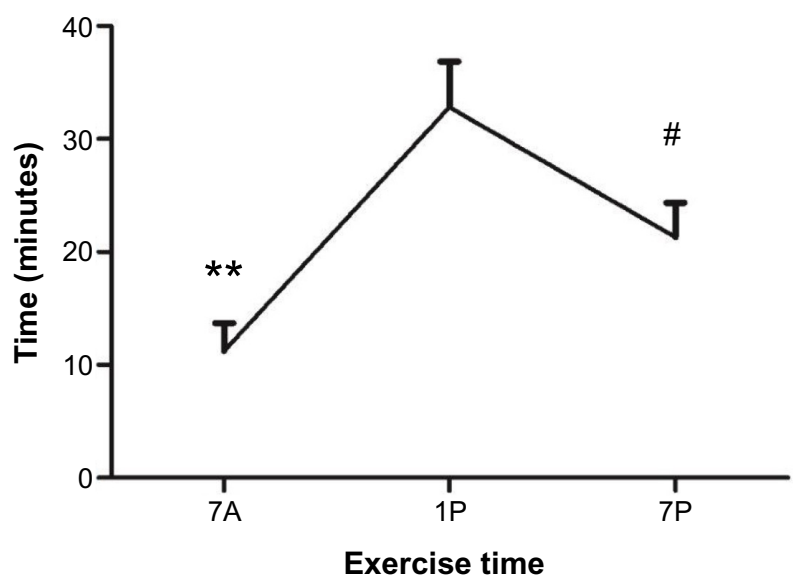

Figure 4 Sleep-onset latency compared to each time-of-day exercise bout. Data are means \pm standard error of mean.

Notes: **7A significantly different from IP and 7P ( $P \leq 0.01)$; "7P significantly different from IP $(P \leq 0.01)$.

Abbreviations: 7A, 7 am exercise condition; IP, I pm exercise condition; 7P, 7 pm exercise condition.

factors influence BP change, and it is possible that PEH may explain the decreased nocturnal BP. Previous literature has shown moderate aerobic exercise conducted in the morning results in the highest reactivity of $\mathrm{PEH}$, with a differential of 8-14 mmHg in mean arterial BP from diurnal to nocturnal pressure values. ${ }^{23}$ The effects of $\mathrm{PEH}$ and sleep patterns are not well understood, but limited research has concluded that exercise in the morning produces the largest fluctuation in BP, which may be due to PEH. ${ }^{24}$ Further, a greater magnitude of $\mathrm{PEH}$ has been documented following periods of nocturnal sleep and absent when exercise was completed after daytime sleep. ${ }^{23}$

Vascular shear stress may explain the nocturnal BP dipping following our acute-exercise bouts. A recent study ${ }^{25}$ that investigated the effects of morning aerobic exercise on vascular shear stress reported that brachial shear stress was significantly higher at 8 am following a 30-minute bout of cycling at $70 \% \mathrm{VO}_{2}$ peak than after conducting the same exercise protocol at $2 \mathrm{pm}$. A similar increase in shear stress may contribute to the decreased nocturnal BP values we recorded following aerobic exercise conducted at 7A in our current study due to prolonged PEH. The decreased sheer stress rate at $2 \mathrm{pm}$ may also validate why we recorded a decreased benefit of aerobic exercise conducted at $1 \mathrm{P}$.

Previous literature demonstrates a difference in the $\mathrm{PEH}$ response between dippers and nondippers. Park et $\mathrm{al}^{2}$ investigated the circadian BP difference between dippers and nondippers following aerobic exercise (30 minutes of intermittent training on a motorized treadmill at $50 \% \mathrm{VO}_{2}$ peak) during 
morning (6-8 am) and evening (5-7 pm) exercise sessions. These researchers concluded that aerobic exercise in the evening exhibited a greater reduction in SBP for nondippers and morning exercise produced similar SBP responses for both dippers and nondippers. ${ }^{2}$ Contrary to our investigation, which solely included a dipping, prehypertensive population, Park et al included participants who were hypertensive and on antihypertensive medications that may produce lower BP responses throughout the day and attenuate the overall BP response to exercise.

The deep stages of non-REM sleep represent heightened neurophysiological restoration ${ }^{26}$ and increased physiological recuperation in muscle and tissue. ${ }^{16}$ Since prior amount of time awake is directly related to the amount of deep sleep, controlling for this in the current investigation allowed for the delineation of the effect of the exercise bout on subsequent deep sleep. Sleep is a period of reduced metabolic requirements essential to energy conservation and tissue restoration. Findings have suggested that deep sleep will increase as a result of increased daytime energy expenditure where exercise is the sole stimulus to deplete energy stores. ${ }^{27}$ Likewise, a decrease in deep sleep is observed during periods of restricted energy intake and weight loss. ${ }^{28}$ Exercise increases time in deep sleep, but there are variances in these results based on the type of exercise performed. ${ }^{16,27}$ Previous literature has shown an increase in deep sleep following an acute bout of moderate-intensity exercise during the afternoon hours. ${ }^{27}$ Our results are consistent with similar studies, but analysis of exercise timing revealed that deep sleep was significantly higher following 7A when compared to the $1 \mathrm{P}$ condition. Several physiological factors may have contributed to this finding; however, the limited evidence regarding the biological factors that control deep sleep only allows for discussion of the possible underlying mechanisms to remain purely speculative.

A significant reduction in REM sleep is generally seen following exercise conditions, ${ }^{27,29}$ and this reduction is greater with longer exercise bouts. ${ }^{29}$ The implications of decreased REM sleep in regard to sleep quality remain unclear. The timing of deep sleep is coupled to sleep onset, but the timing of REM sleep is potentially governed by an individual's circadian rhythm. ${ }^{29,30}$ Therefore, exercise may alter and delay the circadian rhythm phases seen in humans. ${ }^{29}$ Alternatively, there may be a compensatory mechanism for the increases in non-REM sleep seen in response to exercise.

SOL drastically shortened at the 7A time point, but was also significantly decreased at $7 \mathrm{P}$ when compared with $1 \mathrm{P}$ (Figure 4). Previous studies have reported inconsistent findings on SOL, mostly when exercise was performed during the late-evening hours. Although Youngstedt et $\mathrm{al}^{29}$ reported an increase in SOL if exercise was performed within 4 hours of bedtime, several other studies have shown a decrease in SOL when exercise at various intensities was performed within this time period. ${ }^{31}$ To our knowledge, no other studies have compared SOL between different exercise time points within a 24-hour period of time. Our results show that aerobic exercise in the morning hours improved SOL more so than evening exercise.

Mechanisms regarding the beneficial effects of exercise on sleep are speculative, but have been linked to energy conservation, tissue restitution, and temperature downregulation. ${ }^{27,31}$ Temperature downregulation from the anterior hypothalamus in response to exercise-induced body heating has been the most accepted hypothesis, ${ }^{31}$ but lacks support in the literature. We speculate that hormonal influences may be responsible for improved sleep quality following a 7A exercise bout when compared to 1P. Dietary changes, energy expenditure, and specific hormonal factors, such as insulin and cortisol expression, alter the release of leptin. Schmid et $\mathrm{al}^{32}$ reported an increase in deep sleep with a concomitant increase in leptin levels, suggesting a possible relationship between leptin and sleep quality. As previously stated, deep sleep correlates with energy intake and energy expenditure, both of which are regulated by circulating levels of leptin. Single bouts of acute exercise have differing effects on leptin concentrations. When trained crew members rowed at maximal intensity for 30 minutes, exercise leptin levels displayed immediate and significant reductions. ${ }^{33}$ Additionally, after trained males underwent a running protocol for 60 minutes at $70 \%$ of their $\mathrm{VO}_{2}$ peak, leptin concentrations were decreased at both 24 hours and 48 hours postexercise. ${ }^{34}$ None of the aforementioned studies reported leptin concentrations throughout the entire day and night following exercise or when compared to a nonexercise day. The secretion of leptin follows a pulsatile fashion, with its highest levels in the evening and early morning hours. Therefore, the timing of exercise may alter the pulsatile secretion of leptin and should be further researched with respect to sleep architecture.

\section{Conclusion}

The present study indicates that partaking in a bout of moderate-intensity aerobic exercise in the early morning (7A) evokes optimal BP changes and results in an improvement in overall sleep architecture. Research demonstrates decreases in the deep stages of sleep, with concomitant 
increases in the lighter stages and a longer SOL correlated with an individual's approach to middle age..$^{35}$ To date, there have been no well-accepted nonpharmacological or pharmacological interventions to increase the amount of deep sleep. Therefore, utilizing aerobic exercise may be a nonpharmacological treatment to attenuate these alterations and improve sleep quality as one ages. The present investigation reports that the timing of exercise is an important aspect to maximize these effects. It seems that the effect of exercise on the various components of sleep architecture is a complicated phenomenon involving several physiological aspects that require further investigation. In conclusion, the early morning (at 7A) may be the most beneficial time of day to engage in aerobic exercise for the improvement of BP and sleep architecture.

\section{Disclosure}

The authors report no conflicts of interest in this work.

\section{References}

1. Ong KL, Cheung BM, Man YB, Lau CP, Lam KS. Prevalence, awareness, treatment, and control of hypertension among United States adults 1999-2004. Hypertension. 2007;49(1):69-75.

2. Park S, Jastremski CA, Wallace JP. Time of day for exercise on blood pressure reduction in dipping and nondipping hypertension. J Hum Hypertens. 2005;19(8):597-605.

3. Pescatello LS, Franklin BA, Fagard R, Farquhar WB, Kelley GA, Ray CA. American College of Sports Medicine position stand. Exercise and hypertension. Med Sci Sports Exerc. 2004;36(3):533-553.

4. Yilmaz MB, Yalta K, Turgut OO, et al. Sleep quality among relatively younger patients with initial diagnosis of hypertension: dippers versus non-dippers. Blood Press. 2007;16(2):101-105.

5. Smolensky MH, Hermida RC, Castriotta RJ, Portaluppi F. Role of sleep-wake cycle on blood pressure circadian rhythms and hypertension. Sleep Med. 2007;8(6):668-680.

6. Loredo JS, Nelesen R, Ancoli-Israel S, Dimsdale JE. Sleep quality and blood pressure dipping in normal adults. Sleep. 2004;27(6): 1097-1103.

7. Verdecchia P, Angeli F, Cavallini C. Ambulatory blood pressure for cardiovascular risk stratification. Circulation. 2007;115(16) 2091-2093.

8. Ben-Dov IZ, Kark JD, Ben-Ishay D, Mekler J, Ben-Arie L, Bursztyn M. Predictors of all-cause mortality in clinical ambulatory monitoring: unique aspects of blood pressure during sleep. Hypertension. 2007; 49(6):1235-1241.

9. Ohkubo T, Hozawa A, Yamaguchi J, et al. Prognostic significance of the nocturnal decline in blood pressure in individuals with and without high 24-h blood pressure: the Ohasama study. J Hypertens. 2002;20(11): 2183-2189.

10. Javaheri S, Storfer-Isser A, Rosen CL, Redline S. Sleep quality and elevated blood pressure in adolescents. Circulation. 2008;118(10): 1034-1040.

11. Buxton OM, Marcelli E. Short and long sleep are positively associated with obesity, diabetes, hypertension, and cardiovascular disease among adults in the United States. Soc Sci Med. 2010;71(5):1027-1036.

12. Eguchi K, Hoshide S, Ishikawa S, Shimada K, Kario K. Short sleep duration is an independent predictor of stroke events in elderly hypertensive patients. J Am Soc Hypertens. 2010;4(5):255-262.
13. Eguchi K, Pickering TG, Schwartz JE, et al. Short sleep duration as an independent predictor of cardiovascular events in Japanese patients with hypertension. Arch Intern Med. 2008;168(20):2225-2231.

14. Franklin PJ, Green DJ, Cable NT. The influence of thermoregulatory mechanisms on post-exercise hypotension in humans. J Physiol. 1993;470:231-241.

15. Youngstedt SD, Kline CE. Epidemiology of exercise and sleep. Sleep Biol Rhythms. 2006;4(3):215-221.

16. Kubitz KA, Landers DM, Petruzzello SJ, Han M. The effects of acute and chronic exercise on sleep. A meta-analytic review. Sports Med. Apr 1996;21(4):277-291.

17. Reid KJ, Baron KG, Lu B, Naylor E, Wolfe L, Zee PC. Aerobic exercise improves self-reported sleep and quality of life in older adults with insomnia. Sleep Med. 2010;11(9):934-940.

18. MacDonald JR, MacDougall JD, Hogben CD. The effects of exercise duration on post-exercise hypotension. J Hum Hypertens. 2000;14(2):125-129.

19. Casonatto J, Tinucci T, Dourado AC, Polito M. Cardiovascular and autonomic responses after exercise sessions with different intensities and durations. Clinics. 2011;66(3):453-458.

20. Shambroom JR, Fabregas SE, Johnstone J. Validation of an automated wireless system to monitor sleep in healthy adults. J Sleep Res. 2012;21(2):221-230.

21. Pickering TG. The clinical significance of diurnal blood pressure variations. Dippers and nondippers. Circulation. 1990;81(2):700-702.

22. Bristow JD, Honour AJ, Pickering TG, Sleight P. Cardiovascular and respiratory changes during sleep in normal and hypertensive subjects. Cardiovasc Res. 1969;3(4):476-485.

23. Jones H, George K, Edwards B, Atkinson G. Effects of time of day on post-exercise blood pressure: circadian or sleep-related influences? Chronobiol Int. 2008;25(6):987-998.

24. Kenney MJ, Seals DR. Postexercise hypotension. Key features, mechanisms, and clinical significance. Hypertension. 1993;22(5):653-664.

25. Jones H, Green DJ, George KP, Black MA, Atkinson G. Evidence for a greater elevation in vascular shear stress after morning exercise. Med Sci Sports Exerc. 2009;41(6):1188-1193.

26. Benington JH, Heller HC. Restoration of brain energy metabolism as the function of sleep. Prog Neurobiol. 1995;45(4):347-360.

27. Driver HS, Taylor SR. Exercise and sleep. Sleep Med Rev. 2000; 4(4):387-402.

28. Karklin A, Driver HS, Buffenstein R. Restricted energy intake affects nocturnal body temperature and sleep patterns. Am J Clin Nutr. 1994;59(2):346-349.

29. Youngstedt SD, O'Connor PJ, Dishman RK. The effects of acute exercise on sleep: a quantitative synthesis. Sleep. 1997;20(3):203-214.

30. Lavie P. Ultrashort sleep-wake cycle: timing of REM sleep. Evidence for sleep-dependent and sleep-independent components of the REM cycle. Sleep. 1987;10(1):62-68.

31. Myllymäki T, Kyröläinen H, Savolainen K, et al. Effects of vigorous late-night exercise on sleep quality and cardiac autonomic activity. J Sleep Res. 2011;20(1 Pt 2):146-153.

32. Schmid DA, Wichniak A, Uhr M, et al. Changes of sleep architecture, spectral composition of sleep EEG, the nocturnal secretion of cortisol, $\mathrm{ACTH}, \mathrm{GH}$, prolactin, melatonin, ghrelin, and leptin, and the DEX$\mathrm{CRH}$ test in depressed patients during treatment with mirtazapine. Neuropsychopharmacology. 2006;31(4):832-844.

33. Jürimäe J, Jürimäe T. Leptin responses to short term exercise in college level male rowers. Br J Sports Med. 2005;39(1):6-9.

34. Olive JL, Miller GD. Differential effects of maximal- and moderateintensity runs on plasma leptin in healthy trained subjects. Nutrition. 2001;17(5):365-369.

35. Faubel R, Lopez-Garcia E, Guallar-Castillón P, et al. Sleep duration and health-related quality of life among older adults: a population-based cohort in Spain. Sleep. 2009;32(8):1059-1068. 


\section{Publish your work in this journal}

Vascular Health and Risk Management is an international, peerreviewed journal of therapeutics and risk management, focusing on concise rapid reporting of clinical studies on the processes involved in the maintenance of vascular health; the monitoring, prevention and treatment of vascular disease and its sequelae; and the involvement of

metabolic disorders, particularly diabetes. This journal is indexed on PubMed Central and MedLine. The manuscript management system is completely online and includes a very quick and fair peer-review system, which is all easy to use. Visit http://www.dovepress.com/ testimonials.php to read real quotes from published authors.

Submit your manuscript here: http://www.dovepress.com/vascular-health-and-risk-management-journal 